\title{
Superlinear Kirchhoff-type problems of the fractional $p$-Laplacian without the (AR) condition
}

\author{
Jiabin Zuo ${ }^{1,2^{*}} \mathbb{D}$, Tianqing $A n^{1}$ and Mingwei Li ${ }^{3}$
}

\section{"Correspondence:}

zuojiabin88@163.com

${ }^{1}$ College of Science, Hohai

University, Nanjing, P.R. China

${ }^{2}$ Faculty of Applied Sciences, Jilin Engineering Normal University,

Changchun, P.R. China

Full list of author information is

available at the end of the article

\section{Abstract}

In this paper, we study the following superlinear $p$-Kirchhoff-type equation:

$$
\begin{cases}\mathcal{M}\left(\int_{\mathbb{R}^{2 N}} \frac{|u(x)-u(y)|^{p}}{|x-y|^{N+p s}} d x d y\right)(-\Delta)_{p}^{s} u(x)-\lambda|u|^{p-2} u=g(x, u) & \text { in } \Omega, \\ u=0 & \text { in } \mathbb{R}^{N} \backslash \Omega,\end{cases}
$$

Under suitable assumptions on $g(x, u)$ without the (AR) condition, the existence of infinitely many solutions for the Kirchhoff equation of a fractional $p$-Laplacian is obtained by using the fountain theorem. Our conclusions generalize and extend some existing results.

MSC: 49J35; 35A15; 35S15; 35R11;47G20

Keywords: Fountain theorem; Kirchhoff-type equation; Fractional p-Laplacian; Ambrosetti-Rabinowitz condition

\section{Introduction and the main result}

The Kirchhoff-type equation has wide applications in population dynamics, optimization, anomalous diffusion, continuum mechanics, etc. In recent years, research on this subject has been very active; see, for example, [1-10] and the references therein. This paper discuss the existence of infinitely many weak solutions for the following problem:

$$
\begin{cases}\mathcal{M}\left(\int_{\mathbb{R}^{2 N}} \frac{|u(x)-u(y)|^{p}}{|x-y|^{N+p s}} d x d y\right)(-\triangle)_{p}^{s} u(x)-\lambda|u|^{p-2} u=g(x, u) & \text { in } \Omega, \\ u=0 & \text { in } \mathbb{R}^{N} \backslash \Omega,\end{cases}
$$

where $\Omega \subset \mathbb{R}^{N}$ is a bounded smooth domain, $0<s<1<p<\infty, p s<N$. $\lambda$ is a real parameter and $(-\triangle)_{p}^{s}$ is the fractional $p$-Laplacian operator defined by

$$
(-\triangle)_{p}^{s} u(x)=2 \lim _{\varepsilon \rightarrow 0^{+}} \int_{\mathbb{R}^{N} \backslash B_{\varepsilon}(x)} \frac{|u(x)-u(y)|^{p-2}(u(x)-u(y))}{|x-y|^{N+p s}} d x d y,
$$

where $B_{\varepsilon}(x)=\left\{y \in \mathbb{R}^{n}:|x-y|<\varepsilon\right\}$. The function $\mathcal{M}$ satisfies:

$\left(M_{1}\right) \mathcal{M} \in C\left(\mathbb{R}_{0}^{+}, \mathbb{R}^{+}\right), \inf _{t \in \mathbb{R}_{0}^{+}} \mathcal{M}(t) \geq m_{0}>0, m_{0}$ is a positive constant.

c) The Author(s) 2018. This article is distributed under the terms of the Creative Commons Attribution 4.0 International License (http://creativecommons.org/licenses/by/4.0/), which permits unrestricted use, distribution, and reproduction in any medium, provided you give appropriate credit to the original author(s) and the source, provide a link to the Creative Commons license, and indicate if changes were made. 
$\left(M_{2}\right)$ There exists $\theta \in\left[1, \frac{N}{N-p s}\right)$, such that

$$
\theta \widetilde{\mathcal{M}}(t)=\theta \int_{0}^{t} \mathcal{M}(s) d s \geq \mathcal{M}(t) t, \quad \forall t \in \mathbb{R}_{0}^{+}
$$

In the study of the problems (1.1), the following Ambrosetti-Rabinowitz condition [11] is used widely:

$$
0<\mu G(x, t)=\mu \int_{0}^{t} g(x, \tau) d \tau \leq g(x, t) t, \quad x \in \Omega, t \geq r
$$

where $\mu>p \theta$ and $r>0$.

It is well known that (AR) condition is very important for variational method, but cannot be satisfied in many cases. There have been some contributions attempting to replace this condition by new ones, we can consult the references [12-16]. Motivated by this work, in this paper we investigate the existence of infinitely many solutions of problem (1.1) without the (AR) condition. Our result extends Theorem 1 of [17] and Theorem 1.1 of [18].

We assume that $g: \bar{\Omega} \times \mathbb{R}$ is a continuous function satisfying:

$\left(g_{1}\right)$ There exist constants $1<\eta_{1}<\eta_{2}<\cdots<\eta_{m}<p<\alpha<p_{s}^{*}$, and functions $v_{0}(x) \in$ $L^{\beta}(\Omega)$, where $p_{s}^{*}=\frac{p N}{N-p s}, \frac{1}{\alpha}+\frac{1}{\beta}=1, v_{i}(x) \in L^{\frac{\alpha}{\alpha-\eta_{i}}}(\Omega), i=1, \ldots, m$, and $v_{m+1}>0$ is a constant such that

$$
|g(x, u)| \leq\left|v_{0}(x)\right|+\sum_{i=1}^{m}\left|v_{i}(x)\right||u|^{\eta_{i}-1}+v_{m+1}|u|^{\alpha-1}, \quad(x, u) \in \Omega \times \mathbb{R} .
$$

$\left(g_{2}\right)$ There are two constants $\mu>p \theta$ and $\varpi_{0}>0$ such that

$$
G(x, t)=\int_{0}^{t} g(x, s) d s \leq \frac{1}{\mu} g(x, t) t+\varpi_{0}|t|^{p} \quad \text { for any } x \in \Omega, t \in \mathbb{R} .
$$

$\left(g_{3}\right) \lim _{|t| \rightarrow \infty} \frac{G(x, t)}{|t|^{p \theta}} \rightarrow+\infty$ uniformly for $x \in \Omega$.

$\left(g_{4}\right) g(x, t)$ is odd for $t$, i.e. $g(x,-t)=-g(x, t)$ for any $x \in \Omega$ and $t \in \mathbb{R}$.

Remark (i) Note that condition $\left(g_{2}\right)$ is different from the (AR) condition, and is weaker than the condition of $[17,19]$ and $[18]$.

(ii) The function $g(x, t)=|t|^{p \theta-2} t \ln (1+|t|)$ satisfies the conditions $\left(g_{2}\right)$ and $\left(g_{3}\right)$, but it does not satisfy the (AR) condition.

Now we state our main result.

Theorem 1.1 Set $s \in(0,1), N>$ ps. If $\left(M_{1}\right)-\left(M_{2}\right)$ and $\left(g_{1}\right)-\left(g_{4}\right)$ hold. Then, for any $\lambda \in \mathbb{R}$, the problem (1.1) has infinitely many weak solutions $\left\{u_{k}\right\}$ in $X_{0}$ with unbounded energy.

The definition of weak solution will be given in the next section. The framework of this paper is as follows. In Sect. 2 we give the variational framework. Section 3 verifies the Cerami condition. In Sect. 4, we establish the existence of infinitely many weak solutions for problem (1.1) by the fountain theorem. 


\section{Variational framework}

In this section, we first review some basic variational frameworks and main Lemmas that will be used in the next section for problem (1.1). Denote $W=\mathbb{R}^{2 N} \backslash \mathcal{O}$, where $\mathcal{O}=\mathcal{C}(\Omega) \times$ $\mathcal{C}(\Omega) \subset \mathbb{R}^{2 N}$, and $\mathcal{C}(\Omega)=\mathbb{R}^{N} \backslash \Omega$. Define a normed linear space $X$ by

$$
X=\left\{u \in L^{p}(\Omega) \mid \int_{W} \frac{|u(x)-u(y)|^{p}}{|x-y|^{N+p s}} d x d y<\infty\right\}
$$

with norm

$$
\|u\|_{X}=\|u\|_{L^{p}(\Omega)}+\left(\int_{W} \frac{|u(x)-u(y)|^{p}}{|x-y|^{N+p s}} d x d y\right)^{\frac{1}{p}}
$$

Then $X$ is a normed linear space and $C_{0}^{\infty}(\Omega) \subset X$ (see [10], Lemma 2.1). Define a subspace $X_{0} \subset X$ by

$$
X_{0}=\left\{u \in X: u(x)=0 \text { a.e. in } \mathbb{R}^{N} \backslash \Omega\right\} .
$$

Under the equivalent norm

$$
\|u\|_{X_{0}}=\left(\int_{W} \frac{|u(x)-u(y)|^{p}}{|x-y|^{N+p s}} d x d y\right)^{\frac{1}{p}}, \quad u \in X_{0}
$$

$X_{0}$ is a uniformly convex reflexive Banach space ([10], Lemma 2.4).

Now, we give the definition of weak solutions for problem (1.1).

Definition 2.1 We say that $u \in X_{0}$ is a weak solution of problem (1.1), if

$$
\begin{aligned}
& \mathcal{M}\left(\|u\|_{X_{0}}^{p}\right) \int_{W} \frac{|u(x)-u(y)|^{p-2}(u(x)-u(y))(\phi(x)-\phi(y))}{|x-y|^{N+p s}} d x d y \\
& -\lambda \int_{\Omega}|u(x)|^{p-2} u(x) \phi(x) d x-\int_{\Omega} g(x, u(x)) \phi(x) d x=0, \quad \forall \phi \in X_{0} .
\end{aligned}
$$

Subsequently we review some of the properties of the eigenvalue problem and the spectrum of the operator. Consider the problem:

$$
\begin{cases}(-\triangle)_{p}^{s} u=\lambda_{k}|u|^{p-2} u & \text { in } \Omega, \\ u=0 & \text { in } \mathbb{R}^{N} \backslash \Omega,\end{cases}
$$

there is a divergent positive eigenvalue sequence.

$$
\lambda_{1}<\lambda_{2} \leq \cdots \leq \lambda_{k} \leq \lambda_{k+1} \leq \cdots,
$$

whose eigenvalues are the critical values of the functional

$$
T_{p}(u)=\|u\|_{X_{0}}^{p}, \quad u \in \Sigma=\left\{u \in X_{0}: \int_{\Omega}|u|^{p} d x=1\right\} .
$$


We notice that the first eigenvalue $\lambda_{1}:=\inf _{u \in \Sigma} T_{p}(u)>0$. The corresponding eigenfunctions will be denoted by $e_{j}$. More details can be found in [20].

Let $X_{j}=\operatorname{span}\left\{e_{j}\right\}$, define

$$
X_{0}=\overline{\bigoplus_{i=1}^{\infty} X_{i}}, \quad Y_{k}=\bigoplus_{i=1}^{k} X_{i}, \quad Z_{k}=\overline{\bigoplus_{i=k}^{\infty} X_{i}}, \quad k=1,2, \ldots
$$

And let $W_{k}:=\left\{u \in Y_{k}:\|u\|_{X_{0}} \leq \rho_{k}\right\}, N_{k}:=\left\{u \in Z_{k}:\|u\|_{X_{0}}=\gamma_{k}\right\}$, where $\rho_{k}>\gamma_{k}>0$.

Lemma 2.2 (Fountain theorem, [21]) Consider an even functional $T \in C^{1}\left(X_{0}, \mathbb{R}\right)$. Assume for each $k \in \mathbb{N}$, there exist $\rho_{k}>\gamma_{k}>0$ such that

$$
\begin{aligned}
& \left(\Phi_{1}\right) \quad a_{k}:=\max _{u \in Y_{k},\|u\|_{X_{0}}=\rho_{k}} T(u) \leq 0, \\
& \left(\Phi_{2}\right) \quad b_{k}:=\inf _{u \in Z_{k},\|u\|_{X_{0}}=\gamma_{k}} T(u) \rightarrow+\infty, k \rightarrow+\infty, \\
& \left(\Phi_{3}\right) T \text { satisfies the }(P S)_{c} \text { condition for every } c>0 .
\end{aligned}
$$

Then $T$ has an unbounded sequence of critical values.

Define the energy functional $T: X_{0} \rightarrow \mathbb{R}$ corresponding to the problem (1.1) by

$$
T(u)=I(u)-J(u)-H(u)
$$

where

$$
I(u)=\frac{1}{p} \widetilde{\mathcal{M}}\left(\|u\|_{X_{0}}^{p}\right), \quad J(u)=\frac{\lambda}{p} \int_{\Omega}|u|^{p} d x, \quad H(u)=\int_{\Omega} G(x, u) d x .
$$

Lemma 2.3 ([10]) If $\left(M_{1}\right)$ holds, then $I: X_{0} \rightarrow \mathbb{R}$ is of class $C^{1}\left(X_{0}, \mathbb{R}\right)$, and

$$
\left\langle I^{\prime}(u), \phi\right\rangle=\mathcal{M}\left(\|u\|_{X_{0}}^{p}\right) \int_{W} \frac{|u(x)-u(y)|^{p-2}(u(x)-u(y))(\phi(x)-\phi(y))}{|x-y|^{N+p s}} d x d y
$$

for all $u, \phi \in X_{0}$.

Lemma 2.4 ([22], Lemma 2) Assume that $g$ is a continuous function. Let $\left(g_{1}\right)$ holds, then the functional $H$ is of class $C^{1}\left(X_{0}, \mathbb{R}\right)$, and

$$
\left\langle H^{\prime}(u), \phi\right\rangle=\int_{\Omega} g(x, u) \phi d x
$$

for all $u, \phi \in X_{0}$.

Combining Lemma 2.3 with Lemma 2.4 , we get $T \in C^{1}\left(X_{0}, \mathbb{R}\right)$ and

$$
\begin{aligned}
\left\langle T^{\prime}(u), \phi\right\rangle= & \mathcal{M}\left(\|u\|_{X_{0}}^{p}\right) \int_{W} \frac{|u(x)-u(y)|^{p-2}(u(x)-u(y))(\phi(x)-\phi(y))}{|x-y|^{N+p s}} d x d y \\
& -\lambda \int_{\Omega}|u(x)|^{p-2} u(x) \phi(x) d x-\int_{\Omega} g(x, u(x)) \phi(x) d x=0
\end{aligned}
$$

for any $u, \phi \in X_{0}$. Clearly, weak solutions of problem (1.1) are the critical points of energy functional $T$. 


\section{Verification of compactness conditions}

We firstly state two definitions.

Definition 3.1 $([23,24])$ Let $T \in C^{1}\left(X_{0}, \mathbb{R}\right)$, we say that $\mathrm{T}$ satisfies the $(P S)_{c}$ condition at the level $c \in \mathbb{R}$, if any sequence $\left\{u_{n}\right\}_{n} \subset X_{0}$ such that

$$
T\left(u_{n}\right) \rightarrow c, \quad \sup _{\|\phi\|_{X_{0}=1}}\left\{\left|\left\langle T^{\prime}\left(u_{n}\right), \phi\right\rangle\right|\right\} \rightarrow 0 \quad \text { as } n \rightarrow \infty,
$$

possesses a convergent subsequence in $X_{0}$; $T$ satisfies the (PS) condition if $T$ satisfies the $(P S)_{c}$ for all $c \in \mathbb{R}$.

Definition 3.2 $([25,26])$ Let $T \in C^{1}(X, \mathbb{R})$, we say that $T$ satisfies the $(C e)_{c}$ condition at the level $c \in \mathbb{R}$, if any sequence $\left\{u_{n}\right\}_{n} \subset X_{0}$ such that

$$
T\left(u_{n}\right) \rightarrow c, \quad\left(1+\left\|u_{n}\right\|\right) \sup _{\|\phi\| \|_{0}=1}\left\{\left|\left\langle T^{\prime}\left(u_{n}\right), \phi\right\rangle\right|\right\} \rightarrow 0 \quad \text { as } n \rightarrow \infty,
$$

possesses a convergent subsequence in $X_{0}$; $T$ satisfies the (Ce) condition if $T$ satisfies the $(C e)_{c}$ for all $c \in \mathbb{R}$.

When $T$ fulfills the (AR) condition, we know the corresponding energy functional $T$ fulfills the Palais-Smale compactness assumptions, however we dropped the (AR) condition, we show that $T$ fulfills the (Ce) condition.

Lemma 3.3 Let $g: \bar{\Omega} \times \mathbb{R} \rightarrow \mathbb{R}$ be a continuous function satisfying conditions $\left(M_{1}\right)-\left(M_{2}\right)$ and $\left(g_{1}\right)-\left(g_{3}\right)$. Then $T$ fulfills the $(C e)$ condition at level $c \in \mathbb{R}$.

Proof Set $c \in \mathbb{R}$. Suppose $\left\{u_{n}\right\}$ satisfies

$$
T\left(u_{n}\right) \rightarrow c, \quad\left(1+\left\|u_{n}\right\|\right) \sup _{\|\phi\|_{X_{0}=1}}\left\{\left|\left\langle T^{\prime}\left(u_{n}\right), \phi\right\rangle\right|\right\} \rightarrow 0
$$

as $n \rightarrow \infty$.

Step 1 . We prove the sequence $\left\{u_{n}\right\}$ is bounded in $X_{0}$.

Arguing by contradiction, if $\left\{u_{n}\right\}_{n \in \mathbb{N}}$ is unbounded in $X_{0}$. Up to subsequence, still denoted by $\left\{u_{n}\right\}_{n \in \mathbb{N}}$, suppose

$$
\left\|u_{n}\right\|_{X_{0}} \rightarrow+\infty
$$

It follows from (3.1) and (3.2) that

$$
\sup _{\|\phi\|_{X_{0}=1}}\left\{\left|\left\langle T^{\prime}\left(u_{n}\right), \phi\right\rangle\right|\right\} \rightarrow 0
$$

Thus

$$
\left\|u_{n}\right\|_{X_{0}} \sup _{\|\phi\|_{X_{0}=1}}\left\{\left|\left\langle T^{\prime}\left(u_{n}\right), \phi\right\rangle\right|\right\} \rightarrow 0
$$


as $n \rightarrow \infty$. For any $n \in \mathbb{N}$, we consider $v_{n}:=\frac{u_{n}}{\left\|u_{n}\right\| X_{0}}$, then $\left\|v_{n}\right\|_{X_{0}}=1$, so $\left\{v_{n}\right\}_{n \in \mathbb{N}}$ is bounded in $X_{0}$. Similarly to Lemma 1 in [22]. Going if necessary to a subsequence, there exists $v_{\infty}$ such that

$$
\begin{array}{ll}
v_{n} \rightarrow v_{\infty} & \text { in } L^{p}\left(\mathbb{R}^{N}\right), \\
v_{n} \rightarrow v_{\infty} & \text { in } L^{\alpha}\left(\mathbb{R}^{N}\right), \\
v_{n} \rightarrow v_{\infty} & \text { in } \mathbb{R}^{N},
\end{array}
$$

as $n \rightarrow \infty$. We discuss two cases.

Case $1 . v_{\infty} \equiv 0$.

By $\left(g_{2}\right),\left(M_{2}\right)$ and (3.1)-(3.2), we obtain

$$
\begin{aligned}
& \frac{1}{\left\|u_{n}\right\|_{X_{0}}^{p}}\left(T\left(u_{n}\right)-\frac{1}{\mu} T^{\prime}\left(u_{n}\right) u_{n}\right) \\
& \geq \frac{1}{\left\|u_{n}\right\|_{X_{0}}^{p}}\left(\frac{1}{p} \widetilde{\mathcal{M}}\left(\left\|u_{n}\right\|_{X_{0}}^{p}\right)-\frac{1}{\mu} \mathcal{M}\left(\left\|u_{n}\right\|_{X_{0}}^{p}\right)\left\|u_{n}\right\|_{X_{0}}^{p}\right. \\
& \left.\quad+\lambda\left(\frac{1}{\mu}-\frac{1}{p}\right)\left\|u_{n}\right\|_{p}^{p}-\int_{\Omega}\left(G\left(x, u_{n}(x)\right)-\frac{1}{\mu} g\left(x, u_{n}(x)\right) u_{n}(x)\right) d x\right) \\
& \geq \frac{1}{\left\|u_{n}\right\|_{X_{0}}^{p}}\left(\left(\frac{1}{p \theta}-\frac{1}{\mu}\right) \mathcal{M}\left(\left\|u_{n}\right\|_{X_{0}}^{p}\right)\left\|u_{n}\right\|_{X_{0}}^{p}\right)-\lambda\left(\frac{1}{\mu}-\frac{1}{p}\right) \int_{\Omega} v_{n}^{p} d x-\varpi_{0} \int_{\Omega}\left|v_{n}\right|^{p} d x \\
& \geq m_{0}\left(\frac{1}{p \theta}-\frac{1}{\mu}\right)
\end{aligned}
$$

which implies $0 \geq m_{0}\left(\frac{1}{p \theta}-\frac{1}{\mu}\right)$. This is a contradiction.

Case 2. $v_{\infty} \not \equiv 0$.

Setting $\Omega_{1}=\left\{x \in \Omega: v_{\infty} \neq 0\right\}$, it is easy to see that $\left|\Omega_{1}\right|>0$ and

$$
\left|u_{n}(x)\right|=\left|v_{n}\right|\left\|u_{n}\right\|_{X_{0}} \rightarrow+\infty \quad \text { on } \Omega_{1}
$$

as $n \rightarrow \infty$, thanks to (3.2), (3.7). From (3.1) and (3.2), we get $\frac{T\left(u_{n}\right)}{\left\|u_{n}\right\|_{X_{0}}^{\theta \theta}} \rightarrow 0$, that is,

$$
\begin{aligned}
0= & \lim _{n \rightarrow \infty} \frac{1}{\left\|u_{n}(x)\right\|_{X_{0}}^{p \theta}}\left(\frac{1}{p} \widetilde{\mathcal{M}}\left(\left\|u_{n}\right\|_{X_{0}}^{p}\right)-\frac{\lambda}{p}\left\|u_{n}\right\|_{p}^{p}-\int_{\Omega_{1}} G\left(x, u_{n}\right) d x\right. \\
& \left.-\int_{\Omega \backslash \Omega_{1}} G\left(x, u_{n}\right) d x\right) .
\end{aligned}
$$

Note that

$$
0<\lambda_{1}=\min _{u \in X_{0} \backslash\{0\}} \frac{\int_{\Omega} \frac{\mid u(x)-u(y) p^{p}}{|x-y|^{\mid+p s}} d x d y}{\int_{\Omega}|u(x)|^{p} d x},
$$

which implies

$$
\|u\|_{L^{p}(\Omega)}^{p} \leq \frac{1}{\lambda_{1}}\|u\|_{X_{0}}^{p}
$$


Because of $\left(M_{2}\right)$, we get

$$
\widetilde{\mathcal{M}}(t) \leq \widetilde{\mathcal{M}}(1) t^{\theta}, \quad \forall t \in[1,+\infty) .
$$

By (3.9)-(3.11), we obtain

$$
\begin{aligned}
0= & \lim _{n \rightarrow \infty} \frac{1}{\left\|u_{n}\right\|_{X_{0}}^{p \theta}}\left(\frac{1}{p} \widetilde{\mathcal{M}}\left(\left\|u_{n}\right\|_{X_{0}}^{p}\right)-\frac{\lambda}{p}\left\|u_{n}\right\|_{p}^{p}-\int_{\Omega_{1}} G\left(x, u_{n}(x)\right) d x\right. \\
& \left.-\int_{\Omega \backslash \Omega_{1}} G\left(x, u_{n}(x)\right) d x\right) \\
\leq & \frac{1}{p} \widetilde{\mathcal{M}}(1)-\lim _{n \rightarrow \infty} \frac{1}{\left\|u_{n}\right\|_{X_{0}}^{p \theta}}\left(\frac{\lambda}{p}\left\|u_{n}\right\|_{p}^{p}+\int_{\Omega_{1}} G\left(x, u_{n}(x)\right) d x\right. \\
& \left.+\int_{\Omega_{\backslash \Omega_{1}}} G\left(x, u_{n}(x)\right) d x\right) .
\end{aligned}
$$

When $\lambda \geq 0$, from (A), we have

$$
\begin{aligned}
0 \leq & \frac{1}{p} \widetilde{\mathcal{M}}(1)-\lim _{n \rightarrow \infty} \frac{1}{\left\|u_{n}\right\|_{X_{0}}^{p \theta}}\left(\frac{\lambda}{p}\left\|u_{n}\right\|_{p}^{p}+\int_{\Omega_{1}} G\left(x, u_{n}(x)\right) d x\right. \\
& \left.+\int_{\Omega \backslash \Omega_{1}} G\left(x, u_{n}(x)\right) d x\right) \\
\leq & \frac{1}{p} \widetilde{\mathcal{M}}(1)-\lim _{n \rightarrow \infty} \frac{1}{\left\|u_{n}\right\|_{X_{0}}^{p \theta}}\left(\int_{\Omega_{1}} G\left(x, u_{n}(x)\right) d x\right. \\
& \left.+\int_{\Omega_{\backslash} \Omega_{1}} G\left(x, u_{n}(x)\right) d x\right) .
\end{aligned}
$$

When $\lambda<0$, from (A) and (3.10), we get

$$
\begin{aligned}
0 \leq & \frac{1}{p} \widetilde{\mathcal{M}}(1)-\lim _{n \rightarrow \infty} \frac{1}{\left\|u_{n}\right\|_{X_{0}}^{p \theta}}\left(\frac{\lambda}{p}\left\|u_{n}\right\|_{p}^{p}+\int_{\Omega_{1}} G\left(x, u_{n}(x)\right) d x\right. \\
& \left.+\int_{\Omega_{\backslash \Omega_{1}}} G\left(x, u_{n}(x)\right) d x\right) \\
\leq & \frac{1}{p} \widetilde{\mathcal{M}}(1)-\lim _{n \rightarrow \infty} \frac{\lambda}{p \lambda_{1}\left\|u_{n}\right\|_{X_{0}}^{p \theta-p}}-\lim _{n \rightarrow \infty} \frac{1}{\left\|u_{n}\right\|_{X_{0}}^{p \theta}}\left(\int_{\Omega_{1}} G\left(x, u_{n}(x)\right) d x\right. \\
& \left.+\int_{\Omega_{\backslash \Omega_{1}}} G\left(x, u_{n}(x)\right) d x\right) \\
= & \frac{1}{p} \widetilde{\mathcal{M}}(1)-\lim _{n \rightarrow \infty} \frac{1}{\left\|u_{n}\right\|_{X_{0}}^{p \theta}}\left(\int_{\Omega_{1}} G\left(x, u_{n}(x)\right) d x\right. \\
& \left.+\int_{\Omega_{\backslash}} G\left(x, u_{n}(x)\right) d x\right) .
\end{aligned}
$$

It follows from $\left(g_{3}\right)$ and (3.8) that

$$
\frac{G\left(x, u_{n}(x)\right)}{\left\|u_{n}\right\|_{X_{0}}^{p \theta}}=\frac{G\left(x, u_{n}(x)\right)}{\left|u_{n}(x)\right|^{p \theta}} \frac{\left|u_{n}(x)\right|^{p \theta}}{\left\|u_{n}\right\|_{X_{0}}^{p \theta}}=\frac{G\left(x, u_{n}(x)\right)}{\left|u_{n}(x)\right|^{p \theta}}\left|v_{n}(x)\right|^{p \theta} \rightarrow+\infty \quad \text { a.e. } x \in \Omega_{1}
$$


as $n \rightarrow \infty$. Making use of the Fatou lemma, we obtain

$$
\lim _{n \rightarrow \infty} \int_{\Omega_{1}} \frac{G\left(x, u_{n}(x)\right)}{\left|u_{n}(x)\right|^{p \theta}} d x \rightarrow+\infty
$$

By $\left(g_{3}\right)$, we know

$$
\lim _{t \rightarrow+\infty} G(x, t)=+\infty
$$

uniformly for every $x \in \bar{\Omega}$. Therefore, (3.15) means that there are two positive constants $t_{1}$ and $D$ such that

$$
G(x, t) \geq D
$$

for any $x \in \bar{\Omega}$ and $|t|>t_{1}$. In addition, since the continuity of $G$ on $\bar{\Omega} \times \mathbb{R}$, we get

$$
G(x, t) \geq \min _{(x, t) \in \bar{\Omega} \times\left[-t_{1}, t_{1}\right]} G(x, t), \quad \forall|t| \leq t_{1} .
$$

Hence, in view of (3.16) and (3.17), we have

$$
G(x, t) \geq \min \left\{D, \min _{(x, t) \in \bar{\Omega} \times\left[-t_{1}, t_{1}\right]} G(x, t)\right\}:=\varrho, \quad \forall(x, t) \in \bar{\Omega} \times \mathbb{R} .
$$

By (3.2) and (3.18), we obtain

$$
\lim _{n \rightarrow \infty} \int_{\Omega \backslash \Omega_{1}} \frac{G\left(x, u_{n}(x)\right)}{\left|u_{n}(x)\right|^{p \theta}} d x \geq 0 .
$$

Combining (3.19) and (3.12)-(3.14), we have a contradiction.

Step 2 . We prove $\left\{u_{n}\right\} \rightarrow u$ in $X_{0}$ for some $u$.

Let $K(x-y)=|x-y|^{-N-p s}$. For every fixed $\varphi \in X_{0}$, define

$$
Q_{\varphi}(v)=\int_{\mathbb{R}^{2 N}}|\varphi(x)-\varphi(y)|^{p-2}(\varphi(x)-\varphi(y))(v(x)-v(y) K(x-y) d x d y
$$

for any $v \in X_{0}$. By the Hölder inequality and the continuity of $Q_{\varphi}$, we get

$$
\left|Q_{\varphi}(v)\right| \leq\|\varphi\|_{X_{0}}^{p-1}\|v\|_{X_{0}}, \quad \forall v \in X_{0} .
$$

Since $u_{n} \rightarrow u$ in $X_{0}$, we have

$$
\lim _{n \rightarrow \infty} Q_{u}\left(u_{n}-u\right)=0 .
$$

Obviously, $\left\langle T^{\prime}\left(u_{n}\right), u_{n}-u\right\rangle \rightarrow 0$. Then we have

$$
\begin{aligned}
\left\langle T^{\prime}\left(u_{n}\right), u_{n}-u\right\rangle= & \mathcal{M}\left(\left\|u_{n}(x)\right\|_{X_{0}}^{p}\right) Q_{u_{n}}\left(u_{n}-u\right)-\lambda \int_{\Omega}\left|u_{n}\right|^{p-2} u_{n}\left(u_{n}-u\right) d x \\
& -\int_{\Omega} g\left(x, u_{n}\right)\left(u_{n}-u\right) d x \rightarrow 0
\end{aligned}
$$


as $n \rightarrow \infty$. Due to the reflexivity of $X_{0}$, similarly to Lemma 8 in [28], there is a subsequence, still denoted by $\left\{u_{n}\right\}_{n}$, such that

$$
\begin{array}{ll}
u_{n} \rightarrow u & \text { in } X_{0}, \\
u_{n} \rightarrow u & \text { in } L^{r}(\Omega), 1 \leq r<p_{s}^{*}, \\
u_{n} \rightarrow u & \text { a.e. in } \mathbb{R}^{N}
\end{array}
$$

as $n \rightarrow \infty$. So, $g\left(x, u_{n}\right)\left(u_{n}-u\right) \rightarrow 0$ a.e. in $\Omega$ as $n \rightarrow \infty$. The sequence $\left\{g\left(x, u_{n}\right)\left(u_{n}-u\right)\right\}$ is uniformly bounded and equi-integrable in $L^{1}(\Omega)$. By the Vitali Convergence Theorem (see [27]),

$$
\lim _{n \rightarrow \infty} \int_{\Omega} g\left(x, u_{n}\right)\left(u_{n}-u\right) d x=0
$$

By (3.21), we have

$$
\mathcal{M}\left(\left\|u_{n}(x)\right\|_{X_{0}}^{p}\right) Q_{u_{n}}\left(u_{n}-u\right) \rightarrow 0
$$

as $n \rightarrow 0$. It follows from $\left(M_{1}\right)$,

$$
Q_{u_{n}}\left(u_{n}-u\right) \rightarrow 0
$$

as $n \rightarrow \infty$. Combining (3.20) with (3.22), we get

$$
\left(Q_{u_{n}}\left(u_{n}-u\right)-Q_{u}\left(u_{n}-u\right)\right) \rightarrow 0
$$

as $n \rightarrow 0$. Using the Simon inequalities

$$
\begin{aligned}
& \left(|\varrho|^{p-2} \varrho-|\sigma|^{p-2} \sigma\right) \cdot(\varrho-\sigma) \geq D_{P}|\varrho-\sigma|^{p}, \quad p \geq 2, \\
& \left(|\varrho|^{p-2} \varrho-|\sigma|^{p-2} \sigma\right) \cdot(\varrho-\sigma) \geq \widehat{D_{P}} \frac{|\varrho-\sigma|^{2}}{(|\varrho|+|\sigma|)^{2-p}}, \quad 1<p<2,
\end{aligned}
$$

for all $\varrho, \sigma \in \mathbb{R}^{N}$, where $D_{P}, \widehat{D_{P}}>0$ only rely on $p$.

If $p \geq 2$, By (3.23), for $n$ large enough,

$$
\begin{aligned}
\left\|u_{n}-u\right\|_{X_{0}}^{p} \leq & D_{P} \int_{\mathbb{R}^{2 N}}\left(\left|u_{n}(x)-u_{n}(y)\right|^{p-2}\left(u_{n}(x)-u_{n}(y)\right)\right. \\
& \left.-|u(x)-u(y)|^{p-2}(u(x)-u(y))\right) \\
& \times\left(u_{n}(x)-u(x)-u_{n}(y)+u(y)\right) K(x-y) d x d y \\
= & D_{P}\left(Q_{u_{n}}\left(u_{n}-u\right)-Q_{u}\left(u_{n}-u\right)\right)=o(1) .
\end{aligned}
$$

Then $\left\|u_{n}-u\right\|_{X_{0}}^{p} \rightarrow 0$.

If $1<p<2$, though the Hölder inequality, the Simon inequality, and (3.23), we have

$$
\left\|u_{n}-u\right\|_{X_{0}}^{p} \leq \widehat{D_{P}}\left(Q_{u_{n}}\left(u_{n}-u\right)-Q_{u}\left(u_{n}-u\right)\right)^{\frac{p}{2}}\left(\left\|u_{n}\right\|_{X_{0}}^{p}+\|u\|_{X_{0}}^{p}\right)^{\frac{2-p}{2}}
$$




$$
\begin{aligned}
& \leq \widehat{D_{P}}\left(Q_{u_{n}}\left(u_{n}-u\right)-Q_{u}\left(u_{n}-u\right)\right)^{\frac{p}{2}}\left(\left\|u_{n}\right\|_{X_{0}}^{p(2-p) / 2}+\|u\|_{X_{0}}^{p(2-p) / 2}\right) \\
& =C\left(Q_{u_{n}}\left(u_{n}-u\right)-Q_{u}\left(u_{n}-u\right)\right)^{\frac{p}{2}}=o(1),
\end{aligned}
$$

as $n \rightarrow \infty$, where $C>0$. Combining the above two cases, thus, $u_{n} \rightarrow u$ in $X_{0}$. The proof of Lemma 3.3 is completed.

\section{Proof of Theorem 1.1}

Similarly to [5], by a direct calculation, we have the following lemma.

Lemma 4.1 Set $1 \leq q<p_{s}^{*}$ and, for every $k \in \mathbb{N}$, let

$$
\mu_{k}:=\sup \left\{\|u\|_{q}: u \in Z_{k},\|u\|_{X_{0}}=1\right\} .
$$

Then $\mu_{k} \rightarrow 0$ as $k \rightarrow \infty$.

Proof of Theorem 1.1 We only need to verify the conditions $\left(\Phi_{1}\right)$ and $\left(\Phi_{2}\right)$ of Lemma 2.2. Verification of $\left(\Phi_{1}\right)$ : Since $Y_{k}$ is finite dimensional, there exist positive constants $B_{k, q}$ and $\widetilde{B_{k, q}}$ depending on $k, q$, such that for each $u \in Y_{k}$

$$
B_{k, q}\|u\|_{X_{0}} \leq\|u\|_{q} \leq \widetilde{B_{k, q}}\|u\|_{X_{0}}
$$

In view of $\left(g_{3}\right)$, for every $c>\frac{\widetilde{\mathcal{M}}(1)}{p B_{k, p \theta}^{p \theta}}$, there exists $\delta_{c}>0$ such that

$$
G(x, t) \geq c|t|^{p \theta}
$$

for all $x \in \bar{\Omega},|t|>\delta_{c}$. According to the Weierstrass theorem, we get

$$
G(x, t) \geq m_{c}:=\min _{x \in \bar{\Omega},|t| \leq \delta_{c}} G(x, t)
$$

for any $|t| \leq \delta_{c}$. We claim that $m_{c} \leq 0$, since $G(x, 0)=0$ for all $x \in \bar{\Omega}$. Therefore, by (4.2) and (4.3), we obtain

$$
G(x, t) \geq c|t|^{p \theta}-H_{c}
$$

for all $(x, t) \in \bar{\Omega} \times \mathbb{R}$, for suitable positive constant $H_{c} \geq c \delta_{c}-m_{c}$.

By (4.1), (4.4) and (3.11), we get

$$
\begin{aligned}
T(u) & \leq \frac{1}{p} \widetilde{\mathcal{M}}\left(\|u\|_{X_{0}}^{p}\right)-\frac{\lambda}{p}\|u\|_{p}^{p}-c\|u\|_{p \theta}^{p \theta}+H_{c}|\Omega| \\
& \leq \frac{\widetilde{\mathcal{M}}(1)}{p}\|u\|_{X_{0}}^{p \theta}-\frac{\lambda}{p}\|u\|_{p}^{p}-c\|u\|_{p \theta}^{p \theta}+H_{c}|\Omega| \\
& \leq \frac{\widetilde{\mathcal{M}}(1)}{p}\|u\|_{X_{0}}^{p \theta}-c B_{k, p \theta}^{p \theta}\|u\|_{X_{0}}^{p \theta}-\frac{\lambda}{p} B_{k, p}^{p}\|u\|_{X_{0}}^{p}+H_{c}|\Omega| .
\end{aligned}
$$

So, we have, for any $u \in Y_{k}$ with $\|u\|_{X_{0}}=\rho_{k} \geq 1$ for big enough, such that $T \leq 0$. The condition $\left(\Phi_{1}\right)$ holds. 
Verification of $\left(\Phi_{2}\right)$ : There exists $\gamma_{k}>0$ such that

$$
b_{k}:=\inf \left\{T(u): u \in Z_{k},\|u\|_{X_{0}}=\gamma_{k}\right\} \rightarrow+\infty
$$

as $k \rightarrow+\infty$. It follows from $\left(g_{1}\right),\left(M_{1}\right)$ and the Hölder inequality that

$$
\begin{aligned}
T(u) \geq & \frac{1}{p} \widetilde{\mathcal{M}}\left(\|u\|_{X_{0}}^{p}\right)-\frac{\lambda}{p}\|u\|_{p}^{p}-\left\|v_{0}\right\|_{\beta}\|u\|_{\alpha}-\sum_{i=1}^{m}\left\|v_{i}(x)\right\|_{\frac{\alpha}{\alpha-\eta_{i}}}\|u\|_{\alpha}^{\eta_{i}}-v_{m+1}\|u\|_{\alpha}^{\alpha} \\
\geq & C_{K, \lambda}\|u\|_{X_{0}}^{p}-\left\|v_{0}\right\|\left\|\frac{u}{\|u\|_{X_{0}}}\right\|\left\|_{\alpha}\right\| u \|_{X_{0}} \\
& -\sum_{i=1}^{m}\left\|v_{i}(x)\right\|_{\frac{\alpha}{\alpha-\eta_{i}}}\left\|\frac{u}{\|u\|_{X_{0}}}\right\|_{\alpha}^{\eta_{i}}\|u\|_{X_{0}}^{\eta_{i}}-v_{m+1}\left\|\frac{u}{\|u\|_{X_{0}}}\right\|_{\alpha}^{\alpha}\|u\|_{X_{0}}^{\alpha} \\
\geq & C_{K, \lambda}\|u\|_{X_{0}}^{p}-\mu_{k}\left\|v_{0}\right\|_{\beta}\|u\|_{X_{0}}-\sum_{i=1}^{m}\left\|v_{i}(x)\right\|_{\frac{\alpha}{\alpha-\eta_{i}}} \mu_{k}^{\eta_{i}}\|u\|_{X_{0}}^{\eta_{i}}-v_{m+1} \mu_{k}^{\alpha}\|u\|_{X_{0}}^{\alpha},
\end{aligned}
$$

where $\mu_{k}$ is defined in Lemma 4.1 and

$$
C_{K, \lambda}= \begin{cases}\frac{1}{2} m_{0} & \text { if } \lambda \leq 0, \\ \frac{1}{2}\left(m_{0}-\frac{\lambda}{\lambda_{1}}\right) & \text { if } 0<\lambda<\lambda_{1}, \\ \frac{1}{2}\left(m_{0}-\frac{\lambda}{\lambda_{k}}\right) & \text { if } 0<\lambda_{k}<\lambda_{k+1} .\end{cases}
$$

We define $\gamma_{k}$ as

$$
\gamma_{k}=\left(\frac{p C_{K, \lambda}}{\alpha v_{m+1} \mu_{k}^{\alpha}}\right)^{\frac{1}{\alpha-p}} .
$$

Thus $\gamma_{k} \rightarrow+\infty$ as $k \rightarrow \infty$. Note that $\alpha>p$, so for every $\|u\|_{X_{0}}=\gamma_{k}$, we get

$$
\begin{aligned}
T(u) & \geq\|u\|_{X_{0}}^{p}\left(C_{K, \lambda}-v_{m+1} \mu_{k}^{\alpha}\|u\|_{X_{0}}^{\alpha-p}\right)-\mu_{k}\left\|v_{0}\right\|_{\beta} \gamma_{k}-\sum_{i=1}^{m}\left\|v_{i}(x)\right\|_{\frac{\alpha}{\alpha-\eta_{i}}} \mu_{k}^{\eta_{i}} \gamma_{k}^{\eta_{i}} \\
& =\left(1-\frac{p}{\alpha}\right) C_{K, \lambda} \gamma_{k}^{p}-\mu_{k}\left\|v_{0}\right\|_{\beta} \gamma_{k}-\sum_{i=1}^{m}\left\|v_{i}(x)\right\|_{\frac{\alpha}{\alpha-\eta_{i}}} \mu_{k}^{\eta_{i}} \gamma_{k}^{\eta_{i}} \rightarrow+\infty
\end{aligned}
$$

as $k \rightarrow \infty$. Therefore, the condition $\left(\Phi_{2}\right)$ is satisfied. The proof is completed.

\section{Conclusion}

In this article, the existence of infinitely many solutions to Eq. (1.1) is established by using the variational methods (i.e. the fountain theorem). We consider fractional $p$-Kirchhoff problems with more general nonlinearity $g$ in $\Omega$, which improves the previous results. In order to overcome new difficulties, we need to adopt special techniques and methods in our paper. 


\section{Funding}

The work is supported by the Fundamental Research Funds for Central Universities (2017B19714 and 2017B07414), Natural Science Foundation of Jiangsu Province (BK20180500) and Natural Science Foundation of Jilin Engineering Normal University (XYB201814).

\section{Abbreviation}

Not applicable.

Availability of data and materials

Not applicable.

\section{Competing interests}

The authors declare that they have no competing interests.

\section{Authors' contributions}

Each of the authors contributed to each part of this study equally, all authors read and approved the final manuscript.

\section{Author details}

'College of Science, Hohai University, Nanjing, P.R. China. ${ }^{2}$ Faculty of Applied Sciences, Jilin Engineering Normal

University, Changchun, P.R. China. ${ }^{3}$ School of Earth Science and Engineering, Hohai University, Nanjing, P.R. China.

\section{Publisher's Note}

Springer Nature remains neutral with regard to jurisdictional claims in published maps and institutional affiliations.

Received: 21 August 2018 Accepted: 26 November 2018 Published online: 04 December 2018

\section{References}

1. Afrouzi, G.A., Mirzapour, M., Chung, N.T.: Existence and multiplicity of solutions for a $p(x)$-Kirchhoff type equation. Rend. Semin. Mat. Univ. Padova 136, 95-109 (2016)

2. Chen, P., Tang, X.H.: Existence and multiplicity results for infinitely many solutions for Kirchhoff-type problems in RN. Math. Methods Appl. Sci. 37(12), 1828-1837 (2014)

3. Cheng, B.: A new result on multiplicity of nontrivial solutions for the nonhomogeneous Schrödinger-Kirchhoff type problem in RN. Mediterr. J. Math. 13(3), 1099-1116 (2016)

4. Chung, N.T.: Multiple solutions for a $p(x)$-Kirchhoff-type equation with sign-changing nonlinearities. Complex Var Elliptic Equ. 58(12), 1637-1646 (2013)

5. Fiscella, A., Pucci, P.: p-fractional Kirchhoff equations involving critical nonlinearities. Nonlinear Anal., Real World Appl. 35, 350-378 (2017)

6. Caponi, M., Pucci, P.: Existence theorems for entire solutions of stationary Kirchhoff fractional $p$-Laplacian equations. Ann. Mat. Pura Appl. 195(6), 2099-2129 (2016)

7. Mingqi, X., Molica Bisci, G., Tian, G., Zhang, B.: Infinitely many solutions for the stationary Kirchhoff problems involving the fractional $p$-Laplacian. Nonlinearity 29, 357-374 (2016)

8. Pucci, P., Xiang, M.Q., Zhang, B.L.: Multiple solutions for nonhomogeneous Schrödinger-Kirchhoff type equations involving the fractional p-Laplacian in RN. Calc. Var. Partial Differ. Equ. 54(3), 2785-2806 (2015)

9. Xiang, M.Q., Zhang, B.L.: Degenerate Kirchhoff problems involving the fractional $p$-Laplacian without the (AR) condition. Complex Var. Elliptic Equ. 60(9), 1-11 (2015)

10. Xiang, M.Q., Zhang, B.L., Ferrara, M.: Existence of solutions for Kirchhoff type problem involving the non-local fractional p-Laplacian. J. Math. Anal. Appl. 424, 1021-1041 (2015)

11. Ambrosetti, A., Rabinowitz, P.: Dual variational methods in critical point theory and applications. J. Funct. Anal. 14 349-381 (1973)

12. Liu, S.: On superlinear problems without the Ambrosetti and Rabinowitz condition. Nonlinear Anal. 73, 788-795 (2010)

13. Miyagaki, O., Souto, M.: Superlinear problems without Ambrosetti and Rabinowitz growth condition. J. Differ. Equ. 245, 3628-3638 (2008)

14. Costa, D.G., Magalhes, C.A.: Variational elliptic problems which are nonquadratic at infinity. Nonlinear Anal. 23 1401-1412 (1994)

15. Schechter, M., Zou, W.: Superlinear problems. Pac. J. Math. 214, 145-160 (2004)

16. Zou, W.M.: Variant fountain theorems and their applications. Manuscr. Math. 104, 343-358 (2001)

17. Bisci, G.M., Repovs, D., Servadei, R.: Nontrivial solutions of superlinear nonlocal problems. Forum Math. 28, 1095-1110 (2016)

18. Xiang, M., Zhang, B., Guo, X.Y.: Infinitely many solutions for a fractional Kirchhoff type problem via fountain theorem. Nonlinear Anal. 120, 299-313 (2015)

19. Binlin, Z., Bisci, G.M., Servadei, R.: Superlinear nonlocal fractional problems with infinitely many solutions. Nonlinearity 28, 2247-2264 (2015)

20. Servadei, R., Valdinoci, E.: Variational methods for non-local operators of elliptic type. Discrete Contin. Dyn. Syst. 33, 2105-2137 (2013)

21. Bartsch, T.: Infinitely many solutions of a symmetric Dirichlet problem. Nonlinear Anal. 20, 1205-1216 (1993)

22. Thin, N.V.: Nontrivial solutions of some fractional problems. Nonlinear Anal. 38, 146-170 (2017)

23. Struwe, M.: Variational methods, applications to nonlinear partial differential equations and Hamiltonian systems. In: Ergeb. Math. Grenzgeb. Springer, Berlin (1990)

24. Willem, M.: Minimax Theorems. Birkhäuser, Basel (1996) 
25. Cerami, G.: An existence criterion for the critical points on unbounded manifolds. Ist. Lombardo Accad. Sci. Lett. Rend. Sez. A. 112, 332-336 (1978) (in Italian)

26. Cerami, G.: On the existence of eigenvalues for a nonlinear boundary value problem. Ann. Mat. Pura Appl. 124, 161-179 (1980) (in Italian)

27. Rudin, W.: Real and Complex Analysis. McGraw-Hill, New York (1966)

28. Servadei, R., Valdinoci, E.: Mountain pass solutions for non-local elliptic operators. J. Math. Anal. Appl. 389, 887-898 (2012)

Submit your manuscript to a SpringerOpen ${ }^{\circ}$ journal and benefit from:

- Convenient online submission

- Rigorous peer review

- Open access: articles freely available online

- High visibility within the field

- Retaining the copyright to your article

Submit your next manuscript at $\gg$ springeropen.com 